\title{
A New Robust Control Strategy for a Wind Energy Conversion System Based on a T-S Fuzzy Model
}

\author{
Sahbi Abderrahim*‡, Moez Allouche**, Mohamed Chaabane** \\ * Research Laboratory: Numerical Control of Industrial Processes, National School of Engineers of Gabes, University of \\ Gabes, Gabes, 6029, Tunisia. \\ ** Laboratory of Sciences and Techniques of Automatic Control \& Computer Engineering (Lab-STA), National Engineering \\ School of Sfax, Sfax, 3038, Tunisia. \\ (abderrahimsahbi@yahoo.com,moez_allouche@yahoo.fr, Chaabane.ucpi@gmail.com) \\ $+$ \\ Corresponding Author; Sahbi Abderrahim, abderrahimsahbi@yahoo.com
}

Received: 09.05.2020 Accepted:28.06.2020

\begin{abstract}
This paper discusses the design of a robust fuzzy Maximum Power Point Tracking (MPPT) control for a variable speed Wind Energy Conversion System (WECS) powered by a Permanent Magnet Synchronous Generator (PMSG). In fact, the main objective of this research work is to ameliorate the energy conversion efficiency of the WECS in spite the existence of a time-varying wind and model parameter uncertainties, by appropriately regulating the duty cycle of a boost converter used to control the studied device. For this purpose, the Takagi-Sugeno (T-S) fuzzy model with parametric uncertainties is exploited for a fuzzy representation of the nonlinear WECS. Next, a T-S reference model relying on the MPPT algorithm is proposed to rapidly calculate the reference signals. Then, a fuzzy controller is developed to compute the duty cycle needed to ensure a robust tracking and a fast convergence to the maximum power for different wind speed changes. Afterward, the stability analysis of the closed-loop control system is proven by the Lyapunov theory and the $H_{\infty}$ performance. Besides, the controller gains are well determined by solving a Linear Matrix Inequality (LMI) problem. Finally, the robustness and the effectiveness of the suggested fuzzy control scheme are confirmed through both simulation results and experimental tests.
\end{abstract}

Keywords Takagi-Sugeno fuzzy model, Robust control, $H_{\infty}$ performance, Linear Matrix Inequality.

\section{Introduction}

In recent years, the development and the exploitation of the renewable energies have a great progress. This is due to the increasing global electrical energy demand and the worldwide concern for the reduction of carbon dioxide emissions and the environment conservation [1]. More specifically, we mean the wind energy which is considered as one of the most renewable energies used for the production of electrical energy, by reason of its cost-effective, cleanness and reliability.

In light of this, numerous research studies have been focused on extracting the maximum efficiency and, also, ensuring the proper functioning of the WECS. In these studies, different type of wind generator are used [2][3][4].
Precisely, the utilization of the PSMG in the wind application is very convenient thanks to its simplicity, efficiency and reliability especially in the small wind turbine applications $(<20 \mathrm{~kW})$.

Recently, various strategies have been devoted to control the WECS. The classical control approach includes the PI/PID regulators which are the most used due to their easiness and low cost, but these methods remain traditional and present a malfunction of the controller in case of a change in the system parameters [5-6]. The linear robust control techniques [7], the methods based on the Perturb \& Observe (P\&O) [8] and the LQG controllers [9] are extensively used in the wind power optimization problems. Despite the advantages of simplicity, the generated energy shows fluctuation around the maximum operational point 
which causes an energy loss during the transformation phase. Moreover, the nonlinear Backstepping control based on the Lyapunov function [10], first order or high order sliding mode control techniques [11, 12], the neuronal networks approaches [13] and comparison between PID and $\mathrm{H} \infty-$ robust controller [14] have been carried out. Furthermore, an adaptive fuzzy logic control strategy [15], a fuzzy-PI control method to capture the maximum power [16], a pitch angle control based on a neural network [17] and robust nonlinear control of via fuzzy approach [18] are the other control strategies that were utilized.

In the real system operation, there are many sources of uncertainty, disturbance and noise which deteriorate the energy conversion efficiency. Therefore, in this paper; a robust T-S fuzzy control strategy for a WECS is proposed. The suggested control schema requires the use of a boost converter to regulate immediately the duty cycle needed for extracting the maximum power in the presence of model parameter uncertainties and time-varying wind. Here, the Takagi-Sugeno (T-S) fuzzy model with parametric uncertainties is used for modeling the WECS. In addition, a $\mathrm{T}-\mathrm{S}$ reference model relying on the MPPT algorithm is investigated to determine the reference signals corresponding to the maximum power. The stability analysis of the closedloop control system is demonstrated by using the Lyapunov theory and the $H_{\infty}$ performance. Besides, the controller gains are well computed by resolving a Linear Matrix Inequality (LMI). The control performances are thoroughly confirmed by simulation and experimental results.

\section{Wind Energy Conversion System Modeling}

In this section, the suggested device of the PMSG based WECS, see Fig. 1, is detailed. The PSMG is directly coupled to the fixed pitch turbine without gearbox, thus, the generator shaft torque is equal to the turbine mechanical torque. The electrical power produced by the PMSG is rectified by a three -phase Diode Bridge. Meanwhile, the output power obtained is supplied to the resistive load $R_{0}$ via a boost converter. This latter is employed to control the WECS for an MPPT operation, which is done by commanding only one active switch power $S_{H}$, and this reduces the complexity of the control system.

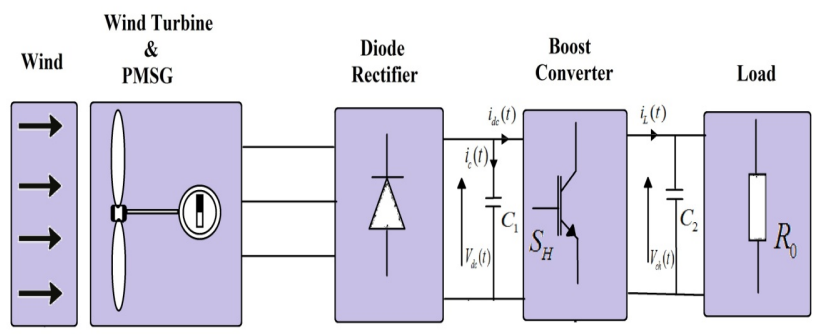

Figure 1 Structural schema of the wind conversion system.
The total power captured from the wind turbine, is stated as [19]:

$$
P_{t}=\frac{1}{2} C_{p}(\lambda, \beta) \rho \pi R^{2} V^{3}
$$

Where $\rho$ is the air density, $R$ is the blade length, $V$ is the wind speed and $C_{p}(\lambda, \beta)$ is the power coefficient defined as follows:

$C_{p}(\lambda, \beta)=0.43\left[\frac{151}{\lambda_{i}}-0.58 \beta-0.002 \beta^{2.14}-13.2\right] \exp \left(\frac{-18.41}{\lambda_{i}}\right)$

with $\lambda_{i}=\left(\frac{1}{\lambda-0.02 \beta}-\frac{0.003}{\beta^{3}+1}\right)^{-1}$

The power coefficient $C_{p}$ depends on the orientation of the blades defined by the pitch angle $\beta$ and the tip speed ratio $\lambda$ which is expressed by the following relation:

$\lambda=\frac{\omega_{t} R}{V}$

Where $\omega_{t}$ represents the mechanical angular speed of the wind turbine.

So as to extract the maximum power from the accessible wind speed, $C_{p}(\lambda, \beta)$ must reach its maximum value. Considering that the blade pitch angle is set to zero $\left(\beta=0^{\circ}\right)$ and according to Eq. 2, $C_{p \max }(\lambda, \beta)$ is reached by maintaining $\lambda$ at its maximum value $\lambda_{\text {opt }}$. Furthermore, $\lambda_{\text {opt }}$ is constant for different wind speeds. Taking Eq.3 into account, and as shown in Fig. 2, there is only one point that can provide the maximum power. Therefore, the wind turbine can be controlled in such a way to track the maximum power point trajectory; this condition is only obtained when the wind turbine speed is always equal to the maximum rotational speed which is expressed as:

$w_{\text {topt }}=\frac{\lambda_{\text {opt }} V}{R}$

Moreover, by using the nonlinear function given in (2), we can determine $C_{p \max }(\lambda, \beta)=0.472$ and $\lambda_{\text {opt }}=8.1$, which enable us to determine the maximum rotational speed $w_{\text {topt }}$ and the maximum power generated by the wind turbine as:

$P_{o p t}=\frac{1}{2} C_{p \max }\left(\lambda_{o p t}, \beta\right) \rho \pi R^{2} V^{3}$

\section{1 wind turbine model}




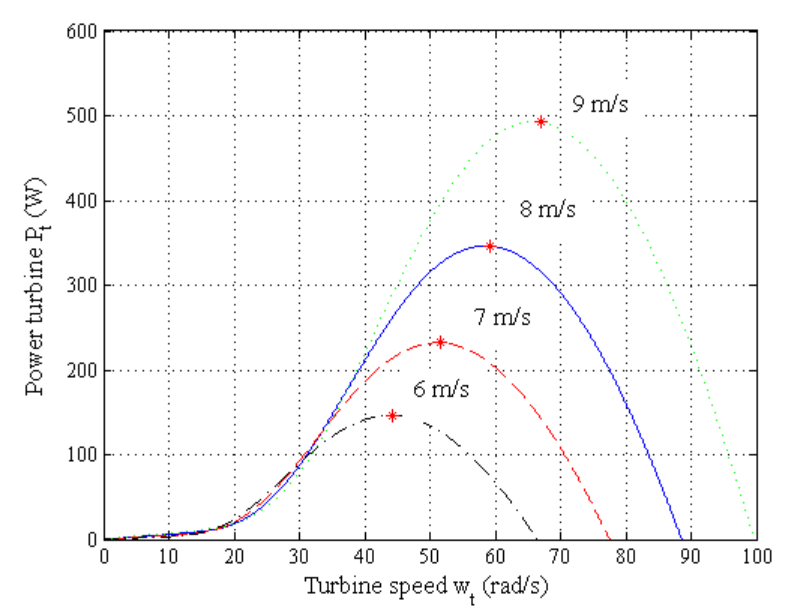

Figure 2 Variation of the generated power versus the turbine speed for different wind speeds.

\subsection{PMSG Model}

Assuming that the mechanical friction has been neglected, the equations of the PMSG can be written in the (d-q) synchronous rotating frame as follows [20]:

$$
\left\{\begin{array}{c}
\frac{d}{d t} i_{s d}=-\frac{R_{s}}{L_{d}} i_{s d}+n_{p} w_{m} i_{s q}+\frac{1}{L_{d}} u_{s d} \\
\frac{d}{d t} i_{s q}=-\frac{R_{s}}{L_{d}} i_{s q}+n_{p} w_{m} i_{s d}-\frac{n_{p}}{L_{d}} w_{m} \phi_{m}+\frac{1}{L_{d}} u_{s q} \\
\frac{d}{d t} w_{m}=\frac{1}{J}\left(C_{t}-C_{e m}\right)
\end{array}\right.
$$

Where $\left(i_{s d}, i_{s q}\right)$ are the stator currents, $\left(u_{s d}, u_{s q}\right)$ are the stator voltages, $J$ is the total moment inertia, $R_{s}$ is the stator resistance, $L d$ is the stator inductance, $w_{m}$ is the rotor speed, $\phi_{m}$ is the flux linked to the magnet, $n_{p}$ is the pole per number of the PMSG and $C_{e m}$ is the electromagnetic torque given as follows:

$C_{e m}=\frac{3}{2} n_{p} i_{s q} \phi_{f}$

The turbine torque is expressed by the following relationship:

$C_{t}=K_{o p t} w_{m}$

Where

$K_{\text {opt }}=0.5 \pi \rho C_{p \max } \frac{R^{5}}{\lambda_{o p t}^{3}}$

The electric power is defined as follows:

$P_{g}=I_{d c} V_{d c}$

Where
$V_{d c}=\frac{3 \sqrt{3}}{\pi} \sqrt{u_{s d}^{2}+u_{s q}^{2}}$ is the DC side voltage resulting from 3-phase rectification and $I_{d c}=\frac{\pi}{2 \sqrt{3}} i_{s q}$ is the DC current. Here, it's important to indicate that the AC/DC converter is perfect and that there is no switching loss power.

\subsection{Boost Converter Model}

The boost converter is associated with the non-controllable rectifier to permit the control of the WECS and to realize the MPPT operation. Furthermore, the maximum power is achieved by adjusting the $S_{H}$ switch duty cycle. Besides, the overall dynamic model of the WECS can be presented by two operational modes of the boost converter depending to the switch power state. Considering the DC- voltage $V_{d c}$, the inductor current $i_{L}(t)$ and the output voltage $V_{c h}$ as state variables, the first mode is determined during the "ON" period as follows:

$$
\left\{\begin{array}{c}
\frac{d V_{d c}(t)}{d t}=-\frac{1}{C_{1}} i_{L}(t)+\frac{1}{C_{1}} i_{d c}(t) \\
\frac{d i_{L}}{d t}=\frac{1}{L} V_{d c}(t)-\frac{R_{L}}{L} i_{L}(t) \\
\frac{d V_{c h}(t)}{d t}=-\frac{1}{R_{0} C_{2}} V_{c h}(t)
\end{array}\right.
$$

and the second mode is calculated during the "OFF" period as follows:

$$
\left\{\begin{array}{c}
\frac{d V_{d c}(t)}{d t}=-\frac{1}{C_{1}} i_{L}(t)+\frac{1}{C_{1}} i_{d c}(t) \\
\frac{d i_{L}(t)}{d t}=\frac{1}{L} V_{d c}(t)-\frac{R_{L}}{L} i_{L}(t)-\frac{1}{L} V_{c h}(t) \\
\frac{d V_{c h}(t)}{d t}=-\frac{1}{R_{0} C_{2}} V_{c h}(t)+\frac{1}{C_{2}} i_{L}(t)
\end{array}\right.
$$

Where $L$ denote the boost inductance, $R_{0}$ is the load resistance, $R_{L}$ is the inductor resistance, $C_{1}$ and $C_{2}$ are the input and the output capacitors, respectively.

Thus, the overall boost converter model can be expressed by the following state space form:

$$
\dot{x}(t)=A_{k} x(t)+E w(t), k=1,2
$$

Where

$$
E=\left[\begin{array}{c}
\frac{1}{C_{1}} \\
0 \\
0
\end{array}\right], w(t)=i_{d c}(t), x(t)=\left[\begin{array}{c}
V_{d c}(t) \\
i_{L}(t) \\
V_{c h}(t)
\end{array}\right]
$$


$A_{1}=\left[\begin{array}{ccc}0 & -\frac{1}{C_{1}} & 0 \\ \frac{1}{L} & -\frac{R_{L}}{L} & 0 \\ 0 & 0 & -\frac{1}{R_{0} C_{2}}\end{array}\right], \quad A_{2}=\left[\begin{array}{ccc}0 & -\frac{1}{C_{1}} & 0 \\ \frac{1}{L} & -\frac{R_{L}}{L} & -\frac{1}{L} \\ 0 & \frac{1}{C_{2}} & -\frac{1}{R_{0} C_{2}}\end{array}\right]$,

Knowing that $u(t) \in[0,1]$ is the duty cycle of the switch power $S_{H}$ during the two operational modes of the boost converter, equation (12) becomes:

$\dot{x}(t)=\left[A_{1} x(t)+E w(t)\right] u(t)+\left[A_{2} x(t)+E w(t)\right](1-u(t))$

Equation (7) can be rewritten as:

$\dot{x}(t)=A_{2} x(t)+\left(A_{1}-A_{2}\right) x(t) u(t)+E w(t)$

Then, we have:

$\dot{x}(t)=A_{2} x(t)+B(x(t)) u(t)+E w(t)$

Where

$$
B(x(t))=\left[\begin{array}{c}
0 \\
\frac{V_{c h}(t)}{L} \\
-\frac{i_{L}(t)}{C_{2}}
\end{array}\right]
$$

\section{Proposed Robust Fuzzy Controller}

This section deals with the robust fuzzy control. The maximum power is achieved by setting the duty cycle of the boost converter proportionally at each wind speed changes. The PMSG coupled with an uncontrolled rectifier is considered as an electrical DC source which supplies a DC load. By this way, the model of the PSMG is not utilized in the control system. Indeed, a fuzzy controller is designed to calculate the duty cycle needed to deliver high performance in spite of the parameter uncertainties and the disturbances caused by the rapid-changing wind speed. Besides, an MPPSearching measure is exploited in the control system to instantly calculate the optimal DC current needed to absorb the maximum generating power. The block diagram of the MPPT robust fuzzy control applied in this paper shows in Fig. 3. The proposed control schema required the measurement of the DC-rectifier voltage $V_{d c}$, the inductor current $i_{L}$ and the output voltage $V_{c h}$, which are compared with their reference value generated via the T-S fuzzy reference model. Thereafter, the T-S fuzzy model, the T-S reference model, the T-S fuzzy uncertain model and the LMI formulation are placed.

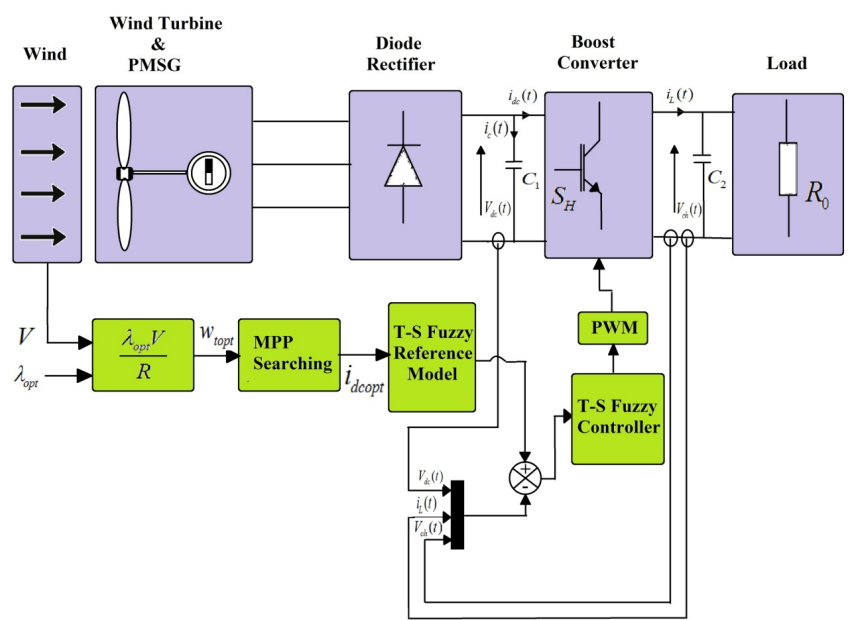

Figure 3 Schematic of the robust control system and its components.

\subsection{T-S Fuzzy modeling of the WECS}

Takagi-Sugeno fuzzy model is an advantageous solution for modeling complex systems, its main is approximating nonlinear dynamics behavior through weighted combination of local affine models interpolated with membership functions. As a matter of fact, the high advantage of this technique is that the stability analysis and the fuzzy controller can be guaranteed by using Lyapunov's theory and parallel distributed compensation (PDC) .[21-22] In this regard, and by observing to the expression (9), the input matrix $B(x(t))$ of the boost converter model contains tow nonlinear terms, can be defined as follows:

$\left\{\begin{array}{l}z_{1}(t)=V_{C 2}(t) \\ z_{2}(t)=I_{L}(t)\end{array}\right.$

And

$$
z_{k}(t)=x_{k}(t) \in\left[\begin{array}{ll}
z_{k \min } & z_{k \max }
\end{array}\right] ; k=1,2
$$

Then, based on the fuzzy premise variables (16), the membership functions take the following form:

$\left\{\begin{array}{l}F_{1, \text { min }}(z(t))=\frac{z_{1}(t)-z_{1, \text { min }}}{z_{1, \text { max }}-z_{1, \text { min }}} \\ F_{1, \text { max }}(z(t))=1-F_{1, \text { min }}(z(t)) \\ F_{2, \text { min }}(z(t))=\frac{z_{2}(t)-z_{2, \text { min }}}{z_{2, \text { max }}-z_{2, \text { min }}} \\ F_{2, \text { max }}(z(t))=1-F_{2, \text { min }}(z(t))\end{array}\right.$

We define the weighting functions as: 


$$
\left\{\begin{array}{l}
h_{1}(z(t))=F_{1, \text { min }}(z(t)) F_{2, \text { min }}(z(t)) \\
h_{2}(z(t))=F_{1, \text { min }}(z(t)) F_{2, \text { max }}(z(t)) \\
h_{3}(z(t))=F_{1, \text { max }}(z(t)) F_{2, \text { min }}(z(t)) \\
h_{4}(z(t))=F_{1, \text { max }}(z(t)) F_{2, \text { max }}(z(t))
\end{array}\right.
$$

Since the T-S fuzzy model has the ability to describe the behavior of a nonlinear system using if-then fuzzy rules; the treated system can be represented by four fuzzy rules:

Rule 1:

$$
\text { If }\left(V_{c h} \text { is } F_{1, \text { min }}\right) \text { and }\left(i_{L} \text { is } F_{2, \text { min }}\right)
$$

Then $\dot{x}(t)=A_{2} x(t)+B_{1} u(t)+E w(t)$

Rule 2:

If $\left(V_{c h}\right.$ is $\left.F_{1, \min }\right)$ and $\left(i_{L}\right.$ is $\left.F_{2, \max }\right)$

Then $\dot{x}(t)=A_{2} x(t)+B_{2} u(t)+E w(t)$

Rule 3:

If $\left(V_{c h}\right.$ is $\left.F_{1, \max }\right)$ and $\left(i_{L}\right.$ is $\left.F_{2, \min }\right)$

Then $\dot{x}(t)=A_{2} x(t)+B_{3} u(t)+E w(t)$

Rule 4:

If $\left(V_{c h}\right.$ is $\left.F_{1, \max }\right)$ and $\left(i_{L}\right.$ is $\left.F_{2, \text { max }}\right)$

Then $\dot{x}(t)=A_{2} x(t)+B_{4} u(t)+E w(t)$

By replacing each of the fuzzy premise variables $z_{k}(t)$ in the input matrix $B(x(t))$ by the corresponding bounded values $\left[\begin{array}{ll}z_{k, \text { min }} & z_{k, \text { max }}\end{array}\right]$, the local subsystem matrices take the following form:

$$
\begin{gathered}
B_{1}=\left[\begin{array}{c}
0 \\
\frac{V_{c h \min }}{L} \\
-\frac{i_{L \min }}{C_{2}}
\end{array}\right] \quad B_{2}=\left[\begin{array}{c}
0 \\
\frac{V_{c h \min }}{L} \\
-\frac{i_{L \max }}{C_{2}}
\end{array}\right] \\
B_{3}=\left[\begin{array}{c}
0 \\
\frac{V_{c h \max }}{L} \\
-\frac{i_{L \min }}{C_{2}}
\end{array}\right] \quad B_{4}=\left[\begin{array}{c}
0 \\
\frac{V_{c h \max }}{L} \\
-\frac{i_{L \max }}{C_{2}}
\end{array}\right]
\end{gathered}
$$

Hence, the inferred output of the fuzzy system is described by:

$$
\dot{x}(t)=\sum_{i=1}^{4} h_{i} z(t)\left(A_{2} x(t)+B_{i} u(t)+E w(t)\right)
$$

Note that the obtained fuzzy model is valid and describes the dynamics behavior of the nonlinear system (15) just under the following operating space:
$\left[\begin{array}{ll}i_{L, \text { min }} & i_{L, \text { max }}\end{array}\right] \times\left[\begin{array}{ll}V_{c h, \text { min }} & V_{c h, \text { max }}\end{array}\right]$

\subsection{Uncertain T-S Fuzzy representation}

To design the robust fuzzy control, the first and the necessary step is to introduce a class of fuzzy systems with uncertainty. On the other hand, the WECS can be affected by a parametric variations caused by the temperature increase and the saturation. Further, the robustness of the proposed fuzzy control schema is evaluaed in terms of maximum power tracking when the nominal value of the internal model parameters varied.

Let's consider an uncertain nonlinear system described by a T-S fuzzy rules as [17]:

Rule $R_{i}$ : If $\left(z_{1}(t)\right.$ is $\left.F_{1 i}\right)$ and $\left(z_{2}(t)\right.$ is $\left.F_{2 i}\right)$ and ....and $\left(z_{m}(t)\right.$ is $\left.F_{m i}\right) \quad$ Then

$x(t)=\left(A_{i}+\Delta A_{i}\right) x(t)+\left(B_{i}+\Delta B_{i}\right) u(t)$

$F_{\kappa i}$ is a fuzzy set $\kappa=1,2, \ldots . m, i=1,2 \ldots . p$

Where $\mathrm{p}$ is the number of the fuzzy rules.

With $\Delta A_{i}$ and $\Delta B_{i}$ denote the uncertainties of the matrix $A_{i}$ and $B_{i}$ respectively and depicted by the following forms:

$$
\left\{\begin{array}{l}
\Delta A_{i}=D_{A i} F_{i}(t) E_{A i} \\
\Delta B_{i}=D_{B i} F_{i}(t) E_{B i}
\end{array}\right.
$$

Where

$D_{(A, B) i}$ and $E_{(A, B) i}$ are real constant matrices with appropriate dimensions. $F_{i}(t)$ is an unknown matrix such that $F_{i}(t) F_{i}^{t}(t)<I$.

Thus, the global uncertain T-S fuzzy system is given by:

$$
\dot{x}(t)=\sum_{i=1}^{m} h_{i}(z(t))\left[\left(A_{i}+\Delta A_{i}\right) x(t)+\left(B_{i}+\Delta B_{i}\right) u(t)\right]
$$

Then, the nonlinear model of the WECS (15) can be expressed by the following uncertain T-S fuzzy representation:

$$
\dot{x}(t)=\sum_{i=1}^{4} h_{i} z(t)\left(A_{2}+\Delta A_{2}\right) x(t)+\left(B_{i}+\Delta B_{i}\right) u(t)+E w(t)
$$

Where

$$
A_{2}+\Delta A_{2}=\left[\begin{array}{ccc}
0 & -\frac{1}{\bar{C}_{1}} & 0 \\
\frac{1}{\bar{L}} & -\frac{\bar{R}_{L}}{\bar{L}} & -\frac{1}{\bar{L}} \\
0 & \frac{1}{\bar{C}_{2}} & -\frac{1}{R_{0} \bar{C}_{2}}
\end{array}\right] B_{i}+\Delta B_{i}=\left[\begin{array}{c}
0 \\
\frac{V_{c h(\min , \max )}}{\bar{L}} \\
-\frac{i_{L(\min , \max )}}{\bar{C}_{2}}
\end{array}\right]
$$


$\bar{C}_{1}=C_{1}+\Delta C_{1}, \bar{C}_{2}=C_{2}+\Delta C_{2}, \bar{L}=L+\Delta L, \bar{R}_{L}=R_{L}+\Delta R_{L}$ are denoted as the uncertainties introduced by system parameters $L, R_{L}, C_{1}$ and $C_{2}$ changed within $20 \%$ of their nominal values, respectively.

\subsection{MPPT algorithm based T-S reference model}

For the purpose of extracting the maximum power under both a model parameter variation and wind speed changes, a MPPT algorithm based T-S reference model is developed. In order to specify the desired trajectories to be tracked. Otherwise, in the steady state, the wind turbine torque is equal to the electromagnetic torque. Thus:

$$
i_{s q}=\frac{2 K_{o p t} w_{m}^{2}}{3 n_{p} \phi_{f}}
$$

Accordingly, the DC current can be expressed in the function of the wind turbine speed which leads us to determine the optimal DC current for converting the maximum power as:

$I_{d c o p t}=\frac{\pi K_{o p t}}{3 \sqrt{3} n_{p} \phi_{f}} w_{\text {mopt }}^{2}$

where

$$
K_{o p t}=0.5 \pi \rho C_{p \max } \frac{R^{5}}{\lambda_{o p t}^{3}}
$$

We mention that the optimal DC current (27) is considered as an input control for the reference model.

In addition, the reference model is chosen in this study is as follows:

$$
\dot{x}(t)=A_{r} x_{r}(t)+r(t)
$$

Where

$A_{r}=\left[\begin{array}{ccc}0 & -\frac{1}{C_{1}} & 0 \\ \frac{1}{L} & -\frac{R_{L}}{L} & -\frac{1}{L}\left(1-u_{o p t}\right) \\ 0 & \frac{1}{C_{2}}\left(1-u_{o p t}\right) & -\frac{1}{R_{0} C_{2}}\end{array}\right], r(t)=\left[\begin{array}{c}\frac{i_{\text {dcopt }}}{C_{1}} \\ 0 \\ 0\end{array}\right], u_{\text {opt }}=1-\sqrt{\frac{V_{\text {dcopt }}}{R_{0} i_{\text {dcopt }}}}$

The reference model contains a non linear term $\left(1-u_{\text {opt }}\right)$ and can be represented using the T-S fuzzy approach via two fuzzy rules as:
Rule 1:

$$
\text { If }\left(z_{r}(t) \text { is } G_{r, \text { min }}\right) \quad \text { Then } \dot{x}(t)=A_{r 1} x_{r}(t)+r(t)
$$

Rule 2:

$$
\text { If }\left(z_{r}(t) \text { is } G_{r, \max }\right) \text { Then } \dot{x}(t)=A_{r 2} x_{r}(t)+r(t)
$$

$\left(G_{r, \min }, G_{r, \max }\right)$ denote the membership functions.

The response of the T-S reference model can be written as follows:

$$
\dot{x}_{r}(t)=\sum_{k=1}^{2} h_{k} z_{r}(t)\left(A_{r, k} x_{r}(t)+r(t)\right)
$$

Where

$$
A_{r 1}=\left[\begin{array}{ccc}
0 & -\frac{1}{C_{1}} & 0 \\
\frac{1}{L} & -\frac{R_{L}}{L} & -\frac{1}{L} z_{r, \min } \\
0 & \frac{1}{C_{2}} z_{r, \min } & -\frac{1}{R_{0} C_{2}}
\end{array}\right], A_{r 2}=\left[\begin{array}{ccc}
0 & -\frac{1}{C_{1}} & 0 \\
\frac{1}{L} & -\frac{R_{L}}{L} & -\frac{1}{L} z_{r, \max } \\
0 & \frac{1}{C_{2}} z_{r, \max } & -\frac{1}{R_{0} C_{2}}
\end{array}\right]
$$

\subsection{LMI Formulation}

In order to reach the maximum power trajectory, the tracking error $e_{r}(t)=x_{r}(t)-x(t)$ must be zero. If this condition is assured, the WECS attain the maximum operational power point. Moreover, the proposed fuzzy tracking control is transformed to a feedback control which easily implemented for a practical system. Hence, the proposed control law is given by:

$u(t)=\sum_{j=1}^{8} h_{j}(z(t)) K_{j}\left(x(t)-x_{r}(t)\right)$

Here, the $H_{\infty}$ performance has been applied to secure a soft reference tracking as [19]:

$$
\begin{aligned}
& \int_{0}^{t f}\left(x_{r}(t)-x(t)\right)^{T} Q\left(x_{r}(t)-x(t)\right) d t \leq \\
& -\gamma^{2} \int_{0}^{t f}\left(r(t) r^{T}(t)+w(t) w^{t}(t)\right) d t
\end{aligned}
$$

Where $\gamma$ is a defined value and $Q$ is a positive defined weighting.

Therefore, by taking the equation (25) into consideration, the dynamic of the tracking error can be deduced as: $\dot{e}_{r}(t)=\sum_{i=1}^{8} \sum_{j=1}^{8} h_{i}(z(t)) h_{j}(z(t))\left(\begin{array}{l}\left(\begin{array}{l}\left(A_{2}+\Delta A_{2}\right) \\ +\left(B_{i}+\Delta B_{i}\right) K_{j}\end{array}\right) e_{r}(t) \\ +\left(A_{2}-A_{r i}+\Delta A_{2}\right) x_{r}(t)\end{array}\right)+E w(t)-r(t)$ 
After that, to design the closed-loop control, an augmented system has been established and is formulated as follows:

$\dot{\bar{x}}=\sum_{i=1}^{8} \sum_{j=1}^{8} h_{i}(z(t)) h_{j}(z(t))\left(\bar{G}_{i j} \bar{x}(t)+\bar{E} \bar{W}(t)\right)$

Where

$\bar{x}(t)=\left(\begin{array}{c}e_{r}(t) \\ x_{r}(t)\end{array}\right)$

$\bar{G}_{i j}=\bar{A}_{i j}+\Delta \bar{A}_{i j}=\left[\begin{array}{cc}\left(A_{2}+B_{i} K_{j}\right) & \left(A_{2}-A_{r i}\right) \\ 0 & A_{r i}\end{array}\right]+\left[\begin{array}{cc}\Delta A_{2}+\Delta B_{i} K_{j} & \Delta A_{2} \\ 0 & 0\end{array}\right]$

$\bar{E}=\left[\begin{array}{cc}E & -I \\ 0 & I\end{array}\right], \bar{W}(t)=\left(\begin{array}{l}w(t) \\ r(t)\end{array}\right)$

By taking equation (34) into account, the $H_{\infty}$ tracking performance (32) can be then modified as follows:

$\int_{0}^{t f}\left(\bar{x}_{r}(t)-\bar{x}(t)\right)^{T} \bar{Q}\left(\bar{x}_{r}(t)-\bar{x}(t)\right) d t \leq$

$-\gamma^{2} \int_{0}^{t f}\left(\bar{w}(t) \bar{w}^{t}(t)\right) d t$

Where

$$
\bar{Q}=\operatorname{diag}\left[\begin{array}{ll}
Q & 0
\end{array}\right]
$$

In fact, the objective of this paper is to design an MPPT fuzzy controller which forces the state variables to track its reference value, in order to carry out a robust tracking performance, a fast convergence and a MPPT operation. In reality, the result of the closed-loop control is deducted in theorem 1 .

Theorem 1 Consider the T-S uncertain fuzzy model (25) regulated by the T-S Fuzzy control law (31), the maximum power is achieved and the Ho performance with the attenuation level $\gamma$ is checked, if there exist some matrices $N_{1}=N_{1}^{T}>0, P_{2}>0$ and a positive scalars $\mu_{1}, \mu_{2}, \mu_{3}$ satisfying the following LMI problem:

$$
\underset{\left(P_{1}, P_{2}\right)}{\min }(\gamma)
$$$$
\left[\begin{array}{ccccccc}
\Pi_{11} & N_{1} & N_{1} E_{a i}^{T} & Y_{j}^{T} E_{b i}^{T} & * & * & * \\
N_{1} & -Q^{-1} & 0 & 0 & 0 & 0 & 0 \\
E_{a i} N_{1} & 0 & -\mu_{2}^{-1} I & 0 & 0 & 0 & 0 \\
E_{b i} Y_{j} & 0 & 0 & -\mu_{3}^{-1} I & 0 & 0 & 0 \\
\left(A_{i}^{T}-A_{r}^{T}\right) & 0 & 0 & 0 & \Pi_{55} & 0 & * \\
I & 0 & 0 & 0 & 0 & -\gamma^{2} I & 0 \\
-I & 0 & 0 & 0 & P_{2} & 0 & -\gamma^{2} I \\
-I & & & & & &
\end{array}\right] \leq 0
$$

Where

$$
\begin{aligned}
& \Pi_{11}=\left(A_{i} N_{1}+B_{i} Y_{j}\right)+\left(A_{i} N_{1}+B_{i} Y_{j}\right)^{T} \\
& +\left(\mu_{1}^{-1}+\mu_{2}^{-1}+\mu_{3}^{-1}\right) H_{i} H_{i}^{t} \\
& \Pi_{55}=A_{r}^{T} P_{2}+P_{2} A_{r}^{T}+\mu_{1} E_{a i}^{T} E_{a i}^{T}
\end{aligned}
$$

Notice: the following symbol $(*)$ indicates the transpose terms in the symmetric position.

The following lemma is utilized in this paper.

Lemma 1: For any matrices $\mathrm{E}$ and $\mathrm{F}$ with appropriate dimension, we have for $\varepsilon>0$ [23]:

$$
E^{T} F+F^{T} E \leq \varepsilon E^{T} E+\varepsilon^{-1} F^{T} F
$$

\section{Proof:}

To perform the stability analysis of the proposed robust fuzzy control, we regard the Lyapunov function depicted by the following equation:

$V(\bar{x}, t)=\bar{x}^{T}(t) \bar{P} \bar{x}(t)$

Next, to ensure a stable closed-loop control based on the $H_{\infty}$ performance, the following inequality must be verified:

$\dot{V}(\bar{x}(t))+\bar{x}^{t}(t) \bar{Q} \bar{x}(t)-\gamma^{2} W^{t}(t) W(t) \leq 0$

By taking equation (37) into consideration, the previous inequality becomes:

$\sum_{i=1}^{8} \sum_{j=1}^{8} h_{i}(z(t)) h_{j}(z(t))\left[\begin{array}{c}\bar{x}(t) \\ W(t)\end{array}\right]^{t}\left[\begin{array}{cc}\bar{G}_{i j} \bar{P}+\bar{P} \bar{G}_{i j}+\bar{Q} & \bar{P} \bar{E} \\ \bar{E} \bar{P} & -\gamma^{2} I\end{array}\right]$

$\left[\begin{array}{c}\bar{x}(t) \\ W(t)\end{array}\right] \leq 0$

Define:

$\bar{P}=\operatorname{diag}\left(P_{1}, P_{2}\right)$

The inequality (39) can be reformulated as follows:

$\left[\begin{array}{cccc}P_{1}\left(A_{i}+\Delta A_{i}+B_{i} K_{j}+\Delta B_{i} K_{j}\right)(*) Q & * & * & * \\ \left(A_{i}^{t}-A_{r}^{t}+\Delta A_{i}^{t}\right) P_{1} & A_{r}^{t} P_{2}+P_{2} A_{r} & 0 & * \\ P_{1} & 0 & -\eta^{2} I & 0 \\ -P_{1} & P_{2} & 0 & -\eta^{2} I\end{array}\right] \leq 0$

In addition, to resolve the inequality (41), we decide to partition it in the following form:

$\bar{\Theta}+\Delta \bar{\Theta} \leq 0$ 
Where

$$
\begin{aligned}
& \bar{\Theta}=\left[\begin{array}{cccc}
P_{1}\left(A_{i}+B_{i} K_{j}\right)+*+Q & * & * & * \\
\left(A_{i}^{t}+A_{r}^{t}\right) P_{1} & A_{r}^{t} P_{2} & 0 & * \\
P_{1} & 0 & -\gamma^{2} I & 0 \\
-P_{1} & P_{2} & 0 & -\gamma^{2} I
\end{array}\right] \\
& \Delta \bar{\Theta}=\left[\begin{array}{cccc}
P_{1}\left(\Delta A_{i}+\Delta B_{i} K_{j}\right)+* & * & 0 & 0 \\
\Delta A_{i}^{T} P_{1} & 0 & 0 & 0 \\
0 & 0 & 0 & 0 \\
0 & 0 & 0 & 0
\end{array}\right]
\end{aligned}
$$

Based on lemma 2, we can eliminate the anti-diagonal elements from the uncertain matrix $\Delta \bar{\Theta}$. Thus, we obtain:

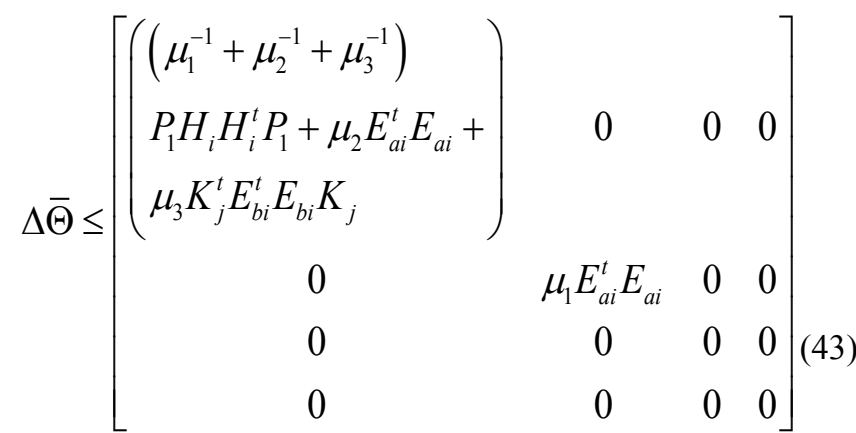

Let's pose:

$$
\begin{aligned}
& \alpha_{i j}=P_{1}\left(A_{i}+B_{i} K_{j}\right)+*+Q \\
& +\left(\mu_{1}^{-1}+\mu_{2}^{-1}+\mu_{3}^{-1}\right) P_{1} H_{i} H_{i}^{t} P_{1} \\
& +\mu_{2} E_{a i}^{t} E_{a i}+\mu_{3} K_{j}^{t} E_{b i}^{t} E_{b i} K_{j}
\end{aligned}
$$

Taking equation (44) into account, the inequality (43) can be reformulated as:

$$
\left[\begin{array}{cccc}
\alpha_{i j} & * & * & * \\
\left(A_{i}^{t}-A_{r}^{t}\right) P_{1} & A_{r}^{t} P_{2}+P_{2} A_{r}+\mu_{1} E_{a i}^{t} E_{a i} & 0 & * \\
P_{1} & 0 & -\gamma^{2} I & 0 \\
-P_{1} & P_{2} & 0 & -\gamma^{2} I
\end{array}\right] \leq 0
$$

After multiplying both of sides of (45) by $\operatorname{diag}\left[\begin{array}{llll}N_{1} & I & I & I\end{array}\right], \quad$ where $\quad P_{1}=N^{-1} \quad$ and $K_{i}=Y_{i} N_{1}^{-1}$, we get:

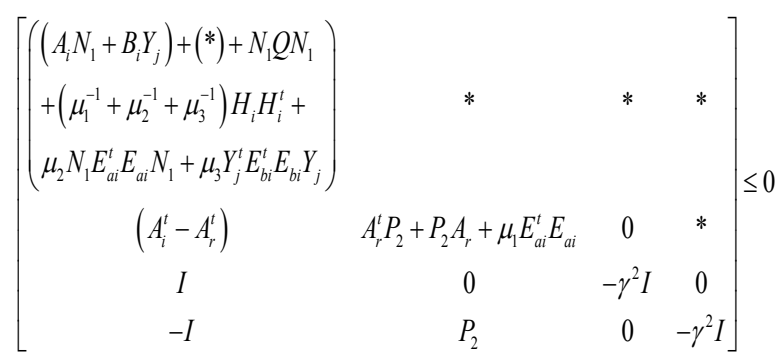

Then, by using the Schur complement to the last inequality, we obtain the standard LMI condition announced in theorem 1 .

\section{Results and discussions}

\subsection{Simulation results}

To verify the robustness of the suggested fuzzy control illustrated in Fig. 3, a simulation result has been performed. The WECS is built using MATLAB/Simulink environment, and its parameters are summarized in Table 1 . The boost converter parameters are given as $L=1 \mathrm{mH}, R_{L}=0.01 \Omega$, $C_{1}=0.001 \mu \mathrm{F}, \quad C_{2}=220 \mu \mathrm{F}$. These parameters are undergoing a variation $20 \%$ of their nominal values. The wind speed used in the simulation tests varies from $4 \mathrm{~m} / \mathrm{s}$ to $8 \mathrm{~m} / \mathrm{s}$, it is shown in Fig. 4 and is defined as [24]:

$$
\begin{aligned}
& V(t)=6.3+0.2 \sin (3.6645 t)+0.5(1.2930 t) \\
& +1.5 \sin (0.2665 t)+0.2 \sin (0.1047 t)
\end{aligned}
$$

The T-S model precisely represents the nonlinear behavior of the WCS (9) if only if $z_{k}(t)$ are chosen as:

$$
z_{1, \text { min }}=0.1 \mathrm{~V} \quad z_{1, \max }=200 \mathrm{~V} \quad z_{2, \min }=-10 \mathrm{~A} \quad z_{2, \max }=10 \mathrm{~A}
$$

By solving the standard LMI formulated in theorem 1, we get the controller gains as follows:

$$
K_{1}=K_{2}=K_{3}=\left[\begin{array}{lll}
-1.3923 & 18.1126 & -1.7841
\end{array}\right]
$$

By applying the T-S fuzzy controller, the evolutions of the rectifier voltage, the inductor current and the output converter voltage are illustrated respectively in Figs. 5, 6 and 7. Similarly, the responses of the duty cycle and the captured power are presented in Figs. 8 and 9.

Table 1. Wind Energy Conversion System parameters

\begin{tabular}{|c|c|c|c|}
\hline Description & Parameters & Value & Units \\
\hline Blade length & $r$ & 1.74 & $\mathrm{~m}$ \\
\hline Stator resistance & $R_{s}$ & 0.57 & $\Omega$ \\
\hline $\begin{array}{c}\text { Stator } \\
\text { inductance } \\
\text { Rotor } \\
\text { inductance }\end{array}$ & $L_{d}$ & 0.0055 & $\mathrm{H}$ \\
\hline $\begin{array}{c}\text { Moment of } \\
\text { inertia }\end{array}$ & $L_{q}$ & 0.0055 & $\mathrm{H}$ \\
\hline $\begin{array}{c}\text { Resistance } \\
\text { No. of pairs }\end{array}$ & $R_{0}$ & 30 & $\Omega$ \\
\hline$n_{p}$ & 4 & - \\
\hline
\end{tabular}




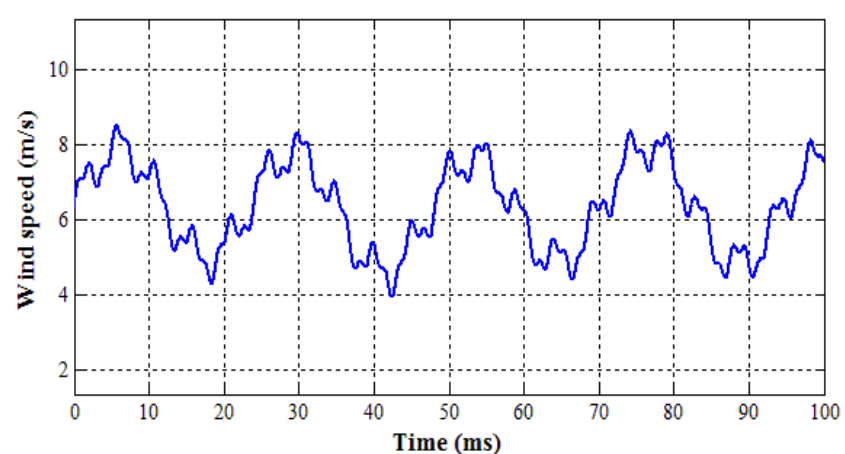

Figure 4 Wind speed profile $(\mathrm{m} / \mathrm{s})$.

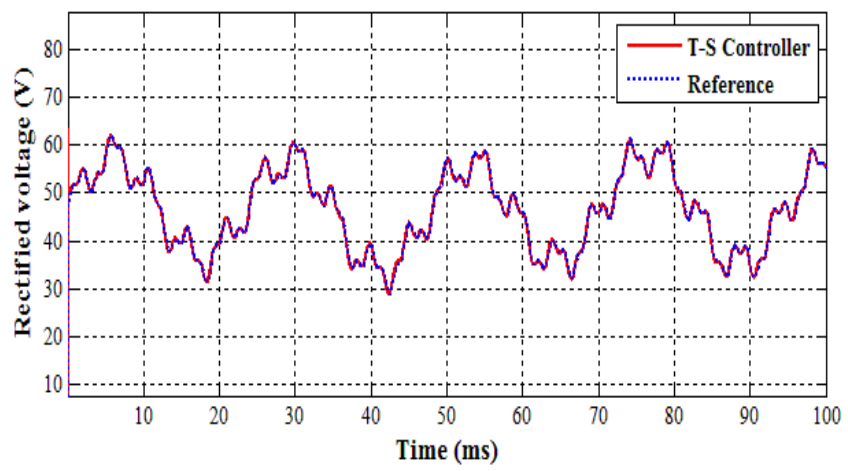

Figure 5 DC rectifier voltage (V).

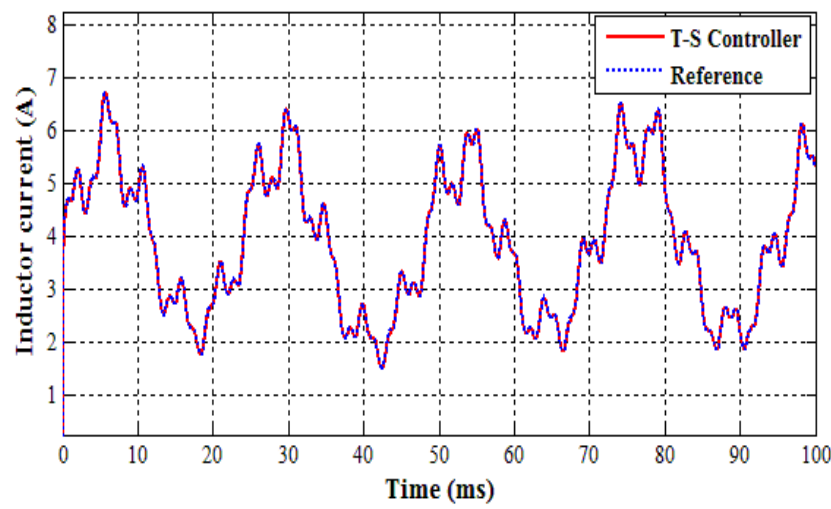

Figure 6 Inductor current (A).

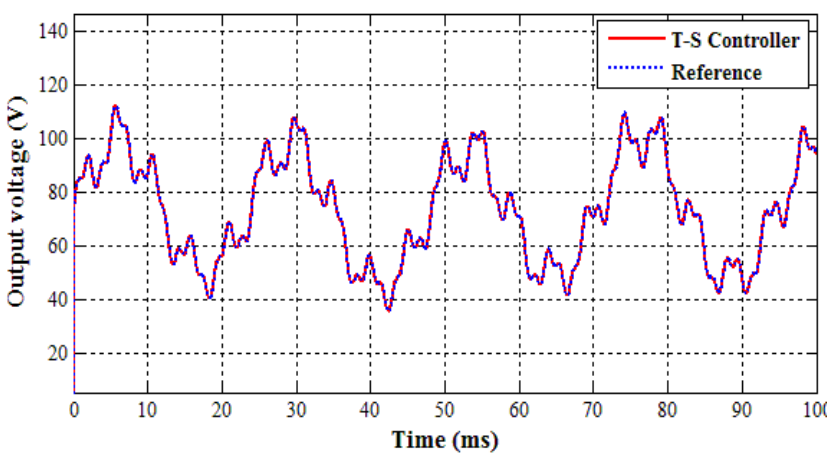

Figure 7 Ouput voltage (V).

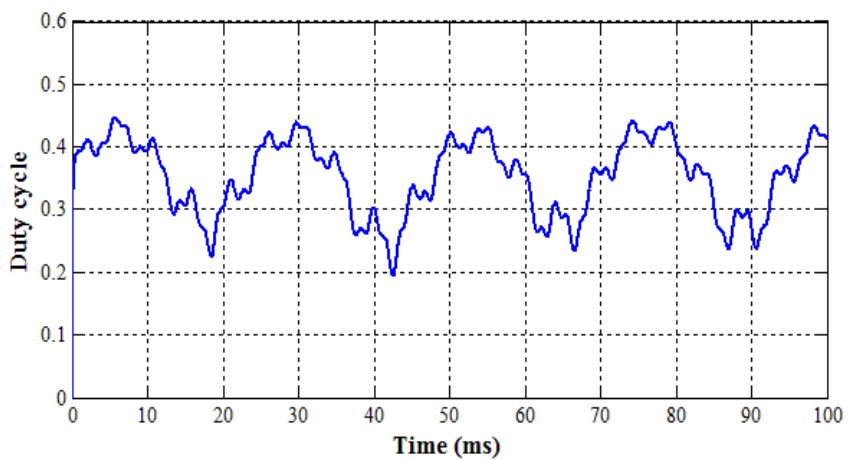

Figure 8 Duty cycle.

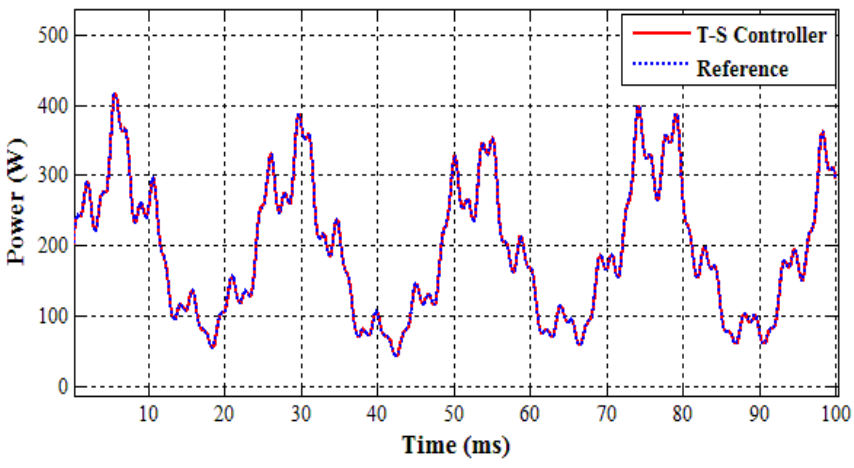

Figure 9 Electrical Power (W).

It can be noticed from Figs 5, 6 and 7 that the T-S fuzzy controller forces the outputs signals of the WECS to track perfectly their reference values corresponding to the maximum power. Therefore, the proposed T-S fuzzy control is robust against the parametric uncertainties and the wind speed changes. Here, the optimal power is achieved by adjusting the duty cycle of the boost converter as shown in Fig. 8 where the duty cycle changes rapidly to insure the MPPT operation. Likewise, the excellent performance of the established control strategy is well illustrated in Fig. 9 where the generating power is equal to the optimal one which means that it ensures the pursuit of the MPP rapidly and precisely. From the simulation results, it is obvious that the suggested control schema has the following highlights:

$>$ a suitable robustness over a wide range of uncertainty up to $20 \%$ and rapidly wind speed variation.

$>$ a rapid speed tracking with high accuracy to the reference values.

$>$ a fast convergence response to the MPP with perfect stabilization.

Consequently, the main objective is excellently achieved since the WECS operates at the MPP and the maximum power is attained in spite of the model parameter uncertainties and wind speed variation. 


\subsection{Experimental validation}

In order to demonstrate the sound performance of the proposed control schema, some experimental tests are carried out. The experimental setup of the WECS is depicted in Figure 10 which was developed in LIAS-Laboratory, France. It is consists of a PMSG directly coupled to a three-phase synchronous motor. This latter is driven by a Leroy Somer industrial speed controller commanded to emulate the behavior of the wind turbine. Besides, based on the wind velocity and the wind turbine power coefficient curve a wind turbine torque has been obtained, which is used as a reference torque to the motor. Furthermore, the three-phase voltage generated by the PSMG is rectified by a three-phase diode bridge coupled to a boost converter made up of a single phase IGBT (SEMIKRON) with anti-paralleling diodes utilized to control the whole system. A LEM PR30 and two HAMEG (HZ64) are used to measure respectively the inductor current, the dc-rectifier voltage and the output voltage. Moreover, the control algorithms constructed using Matlab/Simulink environment and, then, downloaded to the DSPACE (DS1005) processor board which in turn provides the PWM signal to command the boost converter. The PWM frequency is equal to $f=10 \mathrm{kH}$. The measured results and the reference values have been visualize by two oscilloscopes. Indeed, the WECS parameters, the wind profile and the resistance load are similar as in the simulation studies. The wind speed profile is shown in Figure 11. After turning on the WECS prototype at $10 \mathrm{~s}$, the rectified voltage and inductor current with their reference values are given in Figures 12 and 13. The output boost converter voltage, the reference output voltage and the duty cycle are shown in Figure 14. The produced power and the optimal power calculated with MPPT algorithm are illustrated in Figure 15.

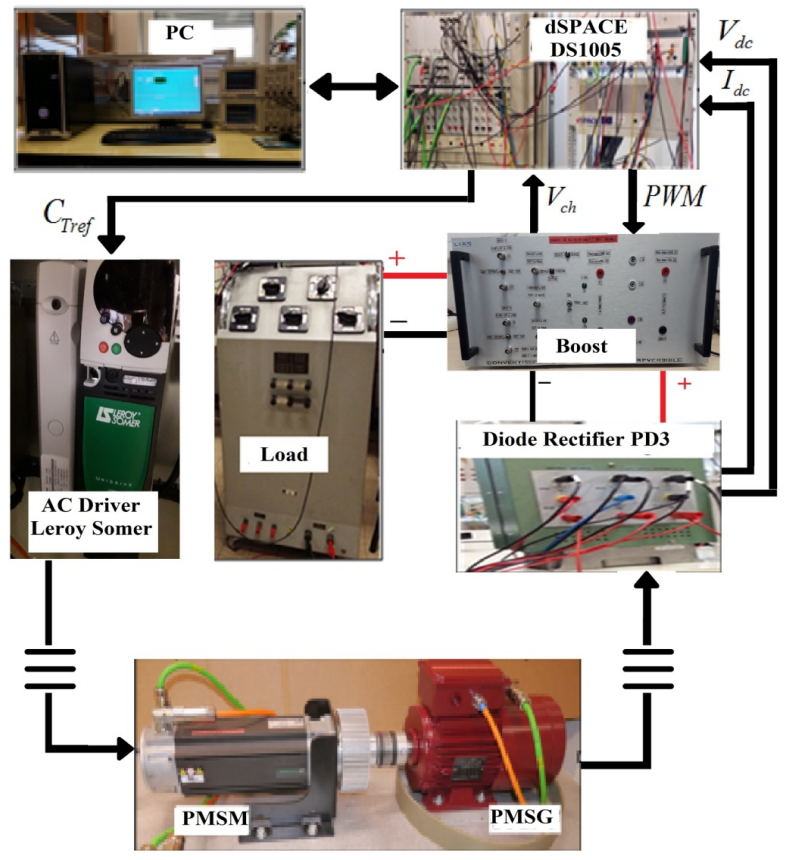

Figure 10 Hardware utilized to co-simulate the WECS.

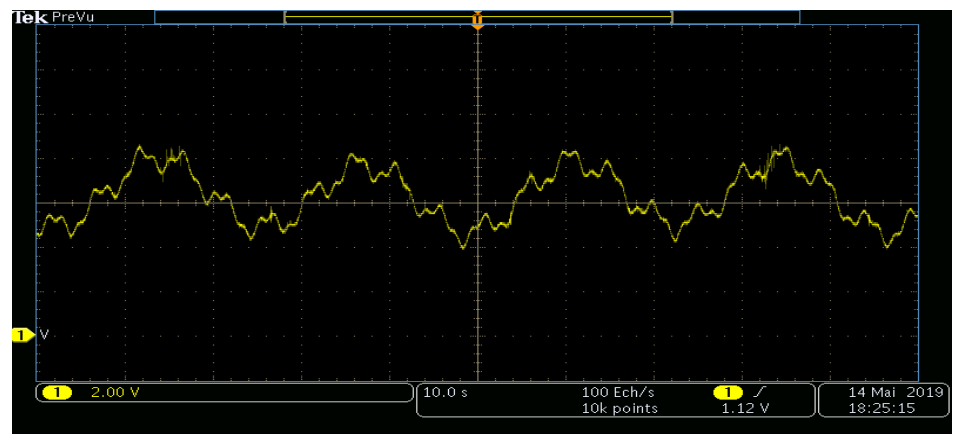

Figure 11 Wind speed variation $(\mathrm{m} / \mathrm{s})$.

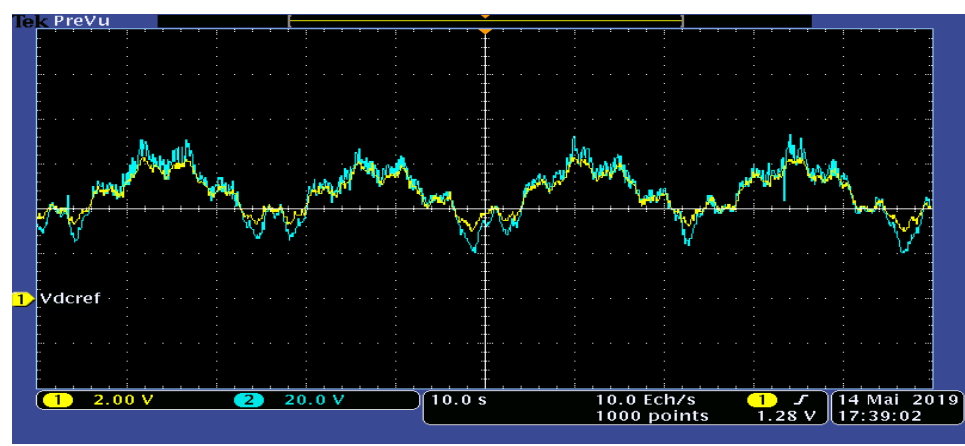

Figure 12 Evolution of the DC rectifier voltage (V)

(obtained voltage and reference voltage).

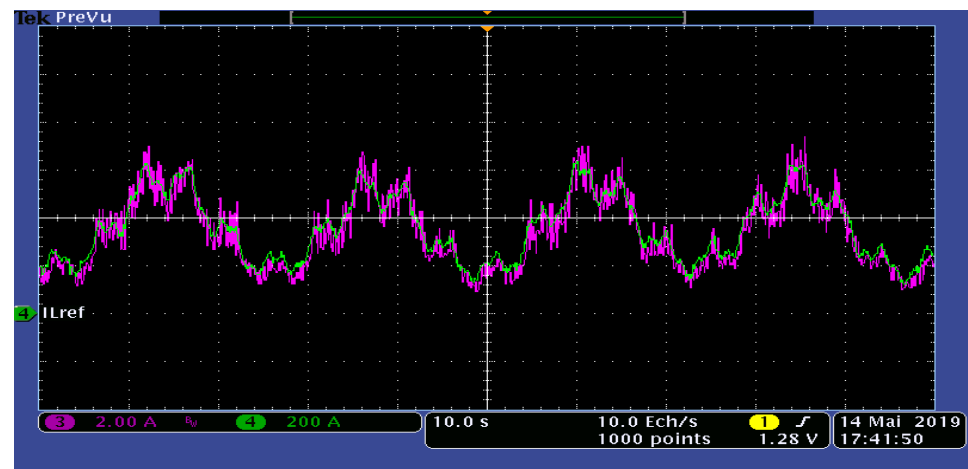

Figure 13 Response the inductor current (A) (controlled current and reference current ).

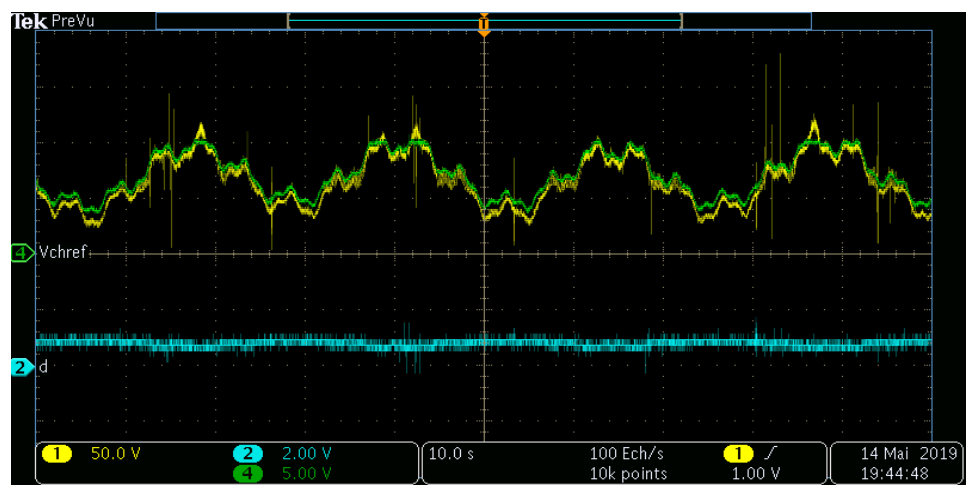

Figure 14 Evolutions of the output boost voltage (V),

(obtained voltage and reference voltage ) and the duty cycle. 


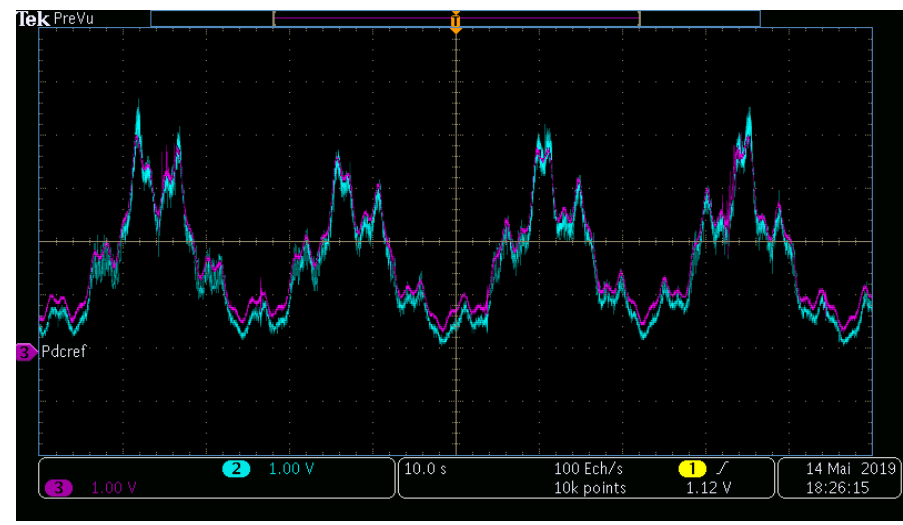

Figure 15 Evolution of the electrical power (W) (response and reference ).

As noticed in the experimental graphs, the same results of the simulation tests have been obtained and this confirms the validity and the effectiveness of the developed approach. Most significantly, the rapid wind speed variation and the parameters uncertainties do not have any effect on the desired performance which proves the robustness of the control system. This is noticeable from Figs. 12, 13 and 14 where the states signals smoothly track their reference signals. Therefore, the main objective of this research work is successfully accomplished since the WECS get at the MPP under all variations in the climatic conditions and the model parameters. This is proven in Fig. 15 in which the producing power is equal to the optimal one.

\section{Conclusion}

This paper proposes a new fuzzy robust control schema for a WECS fed by a PSMG. The main goal of the suggested control strategy is to insure a smooth pursuit of the MPP in the presence of both a time-varying wind and parameter uncertainties. For this, the dynamic behavior of the WECS and the designed controller are well calculated by utilizing the T-S fuzzy approach. Likewise, a T-S reference model based on the MPPT algorithm is established to quickly compute the optimal signals at each wind speedvariation. In addition, the stability condition is proved by means of the Lyapunov theory, and the controller gains are obtained by resolving an optimization problem under LMI constraints. Finally, both simulation and experimental results have given way to show the effectiveness of the proposed design.

\section{Acknowledgements}

Authors may acknowledge to any person, institution or department that supported to any part of study.

\section{References}

[1] C. M. Hong, F. S. Cheng, and C. H. Chen,"Optimal control for variable-speed wind generation systems using general regression neural network", Electrical Power and Energy Systems, vol. 60 , pp.14-23, 2014.
[2] R.J.A. Barrado, J.I. Talpone and L.M. Salamero, "Variable-speed wind energy conversion system based on a dual stator-winding induction generator", IET Renewable Power Generation, vol. 11, pp. 73-80, 2017.

[3] J.L.D. Garcia, O.G. Bellmunt, L.T. Romero and A.J. Ferre, "Indirect vector control of a squirrel cage induction generator wind turbine", Energy Conversion and Management, vol. 64, pp. 102114, 2012.

[4] O. Carranza, E. Figueres, G. Garcera, M.R. Gonzalez and YK Chauhan, "Analysis of the control structure of wind energy generation systems based on a permanent magnet synchronous generator", Applied Energy, vol. 103, pp. 522-538, 2013.

[5] A. Hwas, R. Katebi," Wind Turbine Control Using PI Pitch Angle Controller",IFAC Proceedings, vol. 45. 3, pp. 241-246, 2012.

[6] V. Gassmann, D. Knittel," Hळ-based PI-observers for web tension estimations in industrial unwinding-winding systems", IFAC Proceedings, vol. 41, pp. 1018-1023, 2008.

[7] H. Camblong. "Digital robust control of a variable speed pitch regulated wind turbine for above rated wind speeds", Control Engineering Practice, vol. 16, pp. 946-958, 2008.

[8] K.A. Stol, L.J. Fingersh, "Wind Turbine Field Testing of State-space Control Designs", Technical report, National Renewable Energy Laboratory, 2004.

[9] A. Azizi, H. Nourisola and S. S. Majidabad, "Fault Tolerant Control of Wind Turbines with an Adaptive Output Feedback Sliding Mode Controller", Renewable Energy, vol. 135, pp.5565, 2018.

[10] S. Mensou, A. Essadki, T. Nasser, B.B Idrissi and L. Ben Tarla, “Dspace DS1104 implementation of a robust nonlinear controller applied for DFIG driven by wind turbine, Renewable Energy, vol. 147, pp. 1759-1771, 2020.

[11] J. Mérida, L. T. Aguilar and J. Dávila," Analysis and synthesis of sliding mode control for large scale variable speed wind turbine for power optimization", Renewable Energy, vol. 71, pp. 715-728, 2014.

[12] O. Barambones, "Sliding mode control strategy for wind turbine power maximization", Energies, vol.5, pp. 2310-30, 2012.

[13] A. Dahbi, M. Hachemi, N.N. Said, and M.S.N. Said, "Optimal control for variable-speed wind 
generation systems using General Regression Neural Network", Energy Conversion and Management, vol. 84, pp. 346-353, 2014.

[14] H. Moradi, G. Vossoughi, " Robust control of the variable speed wind turbines in the presence of uncertainties: A comparison between $\mathrm{H} \infty$ and PID controllers ", Energy, pp.1-14,2015.

[15] K. A. Naik, C. P. Gupta and E. Fernandez," Design and implementation of interval type-2 fuzzy logic-PI based adaptive controller for DFIG based wind energy system", Electrical Power and Energy Systems, vol. 115, pp. 1-15, 2020.

[16] A. Beddar, H. Bouzekri, B. Babes, and H. Afghoul, "Experimental enhancement of fuzzy fractional order PI + I controller of grid connected variable speed wind energy conversion system", Energy Conversion and Management, vol. 123, pp. 569-580, 2016.

[17] T.L. Van, T.H. Nguyen, and D.C. Lee,"Advanced Pitch Angle Control Based on Fuzzy Logic for Variable-Speed Wind Turbine Systems", IEEE Transactions on Energy Conversion, vol. 30, pp. $578-587,2015$.

[18] E. Kamal, A. Aitouche, R. Ghorbani, M. Bayart, "Robust nonlinear control of wind energy conversion systems", Electrical Power and Energy Systems, vol. 44, pp.202-209, 2013.

[19] M. Allouche, S. Abderrahim, H. Ben Zina and M. Chaabane, "A Novel fuzzy Control Strategy for Maximum Power Point Tracking of Wind Energy Conversion System", International Journal of Smart Grid, vol. 3, pp.120-127, 2019.

[20] A.M. Shotorbani, B. Mohammadi-Ivatloo, L .Wang, M. Marzband and M. Sabahi,” Application of finitetime control Lyapunov function in lowpower PMSG wind energy conversion systems for sensorless MPPT',Electrical Power and Energy Systems, vol. 106, pp. 169-182, 2019.
[21] A.M. Shotorbani, B. Mohammadi-Ivatloo, L .Wang, M. Marzband and M. Sabahi," Application of finitetime control Lyapunov function in lowpower PMSG wind energy conversion systems for sensorless MPPT",Electrical Power and Energy Systems, vol. 106, pp. 169-182, 2019.

[22] H. Wang, K. Tanaka and M. Griffin, "An approach to fuzzy control of nonlinear systems: Stability and design", IEEE Transactions on Fuzzy Systems, vol. 4, pp. 14-23, 1996.

[23] N. Harrabi, M. Kharrat, A. Aitouche and M. Souissi, "Control Strategies for the Grid Side Converter in a Wind Generation System Based on a Fuzzy Approach", Int. J. Appl. Math. Comput. Sci, vol. 28, pp. 323-333, 2018.

[24] K. Zhou, and P. Khargonedkar, "Robust Stabilization of linear systems with norm-bounded time- varying uncertainty". Sys. Control Letters., vol. 10, pp. 17-20, 1988.

[25] Y. Daili, J.P. Gaubert and L. Rahmani, "Implementation of a new maximum power point tracking control strategy for small wind energy conversion systems without mechanical sensors", Energy Conversion Management, vol. 97, pp. 298306, 2015. 